\title{
Role of PET/CT in muscle-invasive bladder cancer
}

\author{
Seok-ki Kim \\ Department of Nuclear Medicine, National Cancer Center, Goyang-si, Korea \\ Correspondence to: Seok-ki Kim. Department of Nuclear Medicine, National Cancer Center, Goyang-si, Korea. Email: skkim@ncc.re.kr.
}

\begin{abstract}
The purpose of this study covered the diagnostic accuracy and usefulness of positron emission tomography/computed tomography (PET/CT) imaging in muscle invasive bladder cancer patients through previously published literature. Through 30 September, 2019, the PubMed database was searched for eligible articles that evaluated PET/CT imaging in bladder cancer patients. In general, FDG PET/CT, the most commonly used PET/CT imaging, does not show good performance for the detection of primary lesions; however, according to the literature it could accurately assess pelvic lymph node (LN) status better than other imaging technologies and it was especially helpful in determining extra-pelvic recurrences. More recently, non-FDG PET/CT imaging, such as C-11 acetate and C-11 choline, has been introduced. Although further research is required, preliminary results show the potential of these techniques to overcome the drawbacks of FDG. This concise study will overview the role of PET/CT when treating muscle-invasive bladder cancer (MIBC).
\end{abstract}

Keywords: F-18 fluorodeoxyglucose; C-11 acetate; C-11 choline; positron emission tomography/computed tomography (PET/CT); bladder cancer; lymph node (LN); staging; restaging

Submitted Dec 18, 2019. Accepted for publication Mar 12, 2020.

doi: $10.21037 /$ tau.2020.03.31

View this article at: http://dx.doi.org/10.21037/tau.2020.03.31

\section{Introduction}

Bladder cancer is one of the most common urinary tract malignancies, and the majority of cases develop from the uroepithelial tissue. Approximately $30 \%$ of bladder cancers shows muscle-invasive bladder cancer (MIBC), which is relevant to a high risk of distant metastases $(1,2)$, while the remainder are superficial tumors. When diagnosing, about $20-30 \%$ of bladder cancer patients have MIBC (1).

2-Fluorine-18-fluoro-2-deoxy-d-glucose (FDG) is the most common radiotracer for oncologic positron emission tomography (PET). Most malignancies show increased glucose metabolism and intensely accumulate FDG. However, typical urologic malignancies such as prostate cancer and renal cell carcinoma mildly uptake FDG, and this low glucose metabolic rate hampers the role of FDG PET/CT, whereas in the case of bladder cancer, FDG uptake itself is intense unlike other urinary system malignancy. The physiological urinary excretion of FDG masks FDG accumulation of bladder carcinomas (Figure 1). Intense FDG-containing urine that is excreted early, must be flushed using diuresis or delaying acquisition time in order to facilitate the identification of primary bladder cancer and the extravesical spread.

Before any curative treatment is intended, it is necessary to rule out distant metastases. Several regional CT and MRI are the optimal diagnostic techniques to assess lung and liver metastases, respectively. Bone and brain metastases are not common at the time of diagnosing MIBC, and additional skeletal and brain imaging are not routinely indicated without specific symptoms or signs. Metastatic lesions far from the intense FDG-containing urine can be investigated well. Accumulation of evidence in the literature has been suggesting that FDG-PET/CT have clinical potential for staging metastatic bladder cancer; however, currently no consensus has been reached, and further confirmatory studies or trials are necessary for a recommendation.

Until now, reimbursement of FDG PET/CT has been limited in urinary malignancy, and large-scale clinical trials with good evidence are sparse, and studies for bladder cancer are also rare. This short review summarized the role of PET/ CT imaging using the currently available knowledge. 

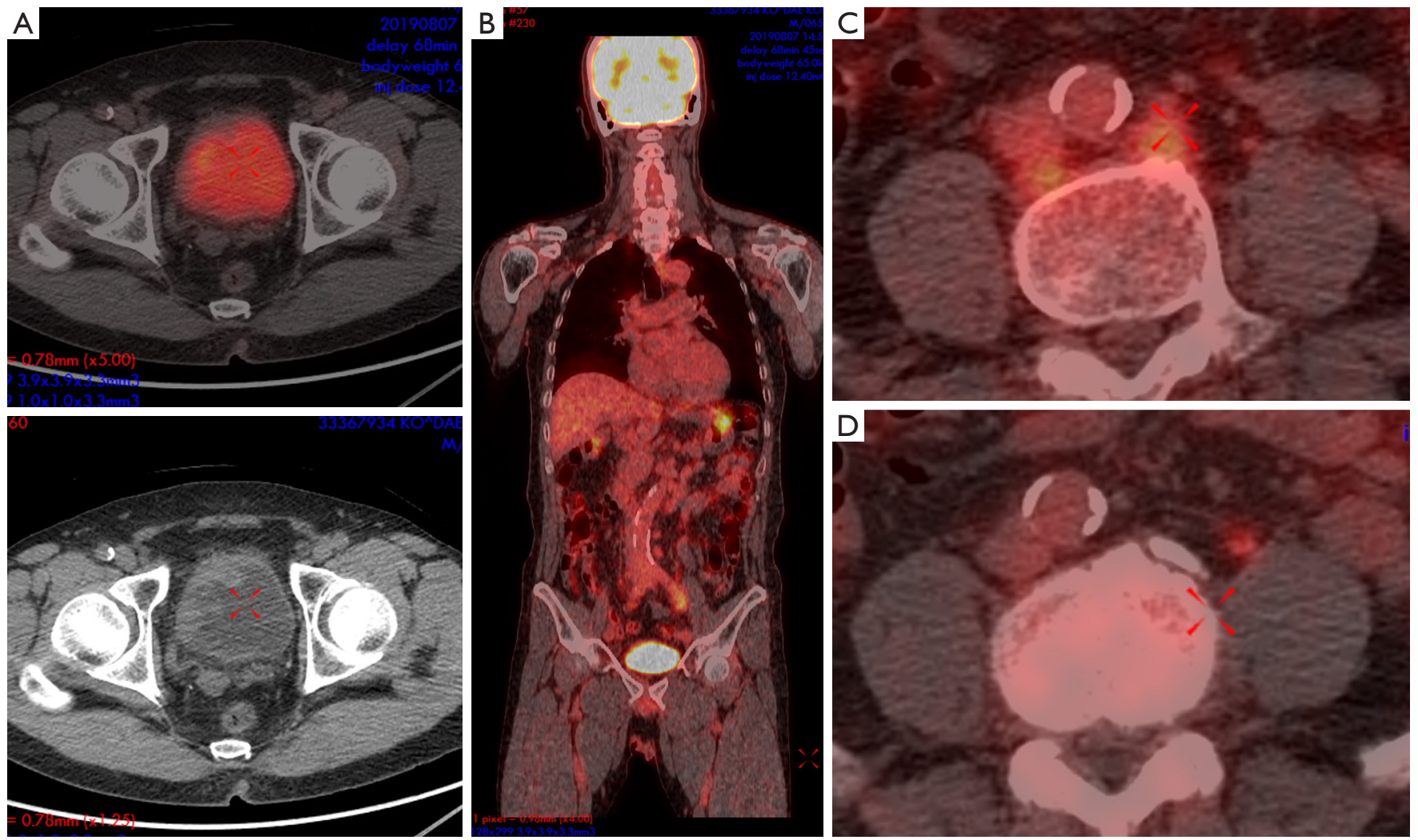

Figure 1 A 65-year-old male with bladder cancer (A). Intense FDG-containing urine obscures FDG uptake in the bladder wall. Histopathologic analysis showed metastatic disease in a left paraaortic lymph node (B,C), which showed F-18 FDG uptake on a PET/ CT, coronal, and axial PET/CT fusion image. After transurethral resection and chemotherapy, F-18 FDG avid lymph node enlargement disappeared (D).

\section{Role of imaging for the staging of MIBC}

The most common symptom of bladder cancer is hematuria. Cystoscopy or renal tract ultrasound is the initial evaluation step, and both cystoscopy and biopsy remain a key step for the investigation of suspicious bladder cancer. The role of MIBC imaging encompasses diagnosis and differential diagnosis, staging of known tumor, and re-staging after recurrence. In the clinic, CT and MRI are the imaging techniques used routinely in bladder cancer, and both $\mathrm{CT}$ and MRI may be used assessing local invasion. The principal reason of CT and MRI is to discover T3b disease or higher because both are unable to accurately detect microscopic invasion of the peri-vesical fat (T2 vs. T3a). The European Association of Urology (3) and the National Comprehensive Cancer Network (NCCN) suggests staging confirmed MIBC with CT or MR of the chest, abdomen, and pelvis, if available (4). Occasionally a bone scintigraphy may be required if there is history, symptom or signs suggesting bony disease.

FDG PET/CT is assumed to be unable to evaluate microscopic peri-vesical fat invasion and adjacent organ involvement. Currently, FDG PET/CT is not recommended as the initial diagnosing or primary staging tool of bladder cancer due to the physiological uptake of FDG in the bladder. In order to overcome these limitations of FDG, profuse water uptake, use of diuretics, and voiding with catheter are known to be helpful (5). According to recent reports, early dynamic FDG images could be helpful for the primary detection of bladder cancer before FDG excrete and collection (6). Several researchers have explored the value of FDG PET or PET/CT when detecting and staging bladder carcinoma, but it remained indecisive and varied. Metaanalysis for diagnostic performance of FDG PET/CT in bladder cancer showed that the pooled sensitivity was 0.82 , 
Table 1 Diagnostic performance of FDG PET/CT in lymph node staging of bladder cancer

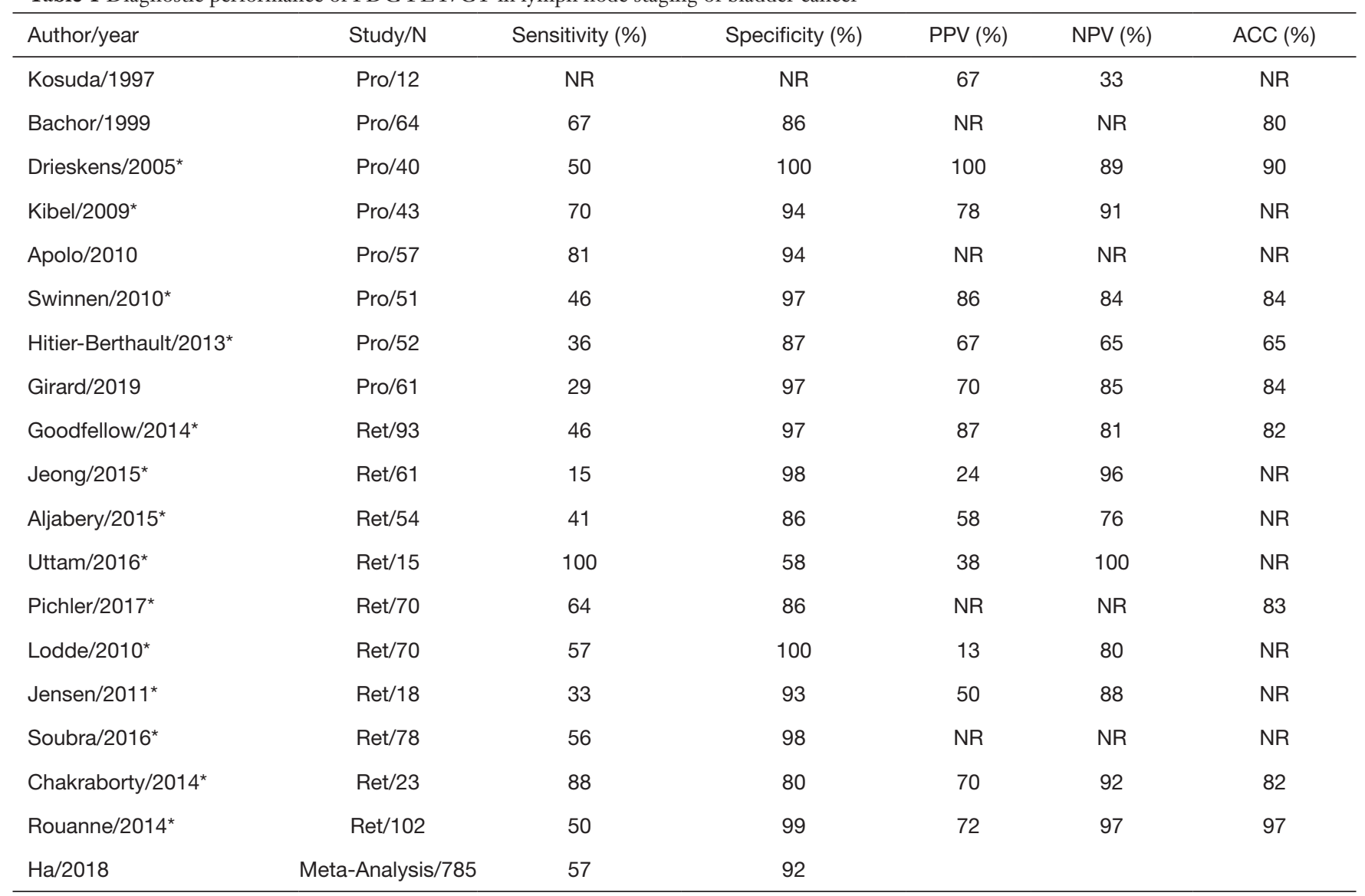

* included in the meta-analysis by Ha HK et al. N, enrolled patients; PPV, Positive Predictive Value; NPV, negative predictive value; ACC, accuracy; Pro, prospective; Ret, retrospective; NR, not reported in the article.

while the specificity was 0.92 in the detection of bladder lesions (7). The analysis included 9 retrospective and 1 prospective study with 433 patients.

\section{Role of FDG PET/CT imaging for lymph nodes (LNs) in MIBC}

A $10-30 \%$ risk of pelvic LN metastasis are known in patients with MIBC. With extending into the peri-vesical fatty tissue, the risk increases up to $50 \%(8,9)$. Assessment of LN metastases is limited on the basis of solely LN size as both CT and MRI could not identify metastases in normalsized or minimally enlarged nodes. The sensitivity for detecting LN metastases is low, as is the specificity since the nodal enlargement may be due to benign disease. Overall, CT and MR are not sufficiently accurate in the evaluation of preoperative LN involvement in bladder cancer $(10,11)$.

Currently, no evidence is supporting that PET can be routinely used in the nodal staging of bladder cancer. Diverse small-scale trials have been widely undertaken using various imaging protocols. Delayed pelvic images after profuse oral hydration and/or diuretics administration are most commonly used among the various techniques that have been studied to improve the diagnostic performances of FDG PET (12-14). There have been several small prospective (12,15-17) and retrospective trials (18-27) for performance staging LNs, the results of which varied widely. The reported sensitivity, specificity, positive predictive value (PPV), negative predictive value (NPV), and accuracy was $15-100 \%, 80-100 \%, 13-100 \%, 33-100 \%$, and $65-97 \%$, respectively (14-16,18-33) (Table 1).

Although in some early studies, FDG PET/CT provided relatively good sensitivity and specificity, a very wide range of sensitivity and specificity was shown among whole studies. Overall, the sensitivity ranges from around 50\% and the specificity around $90 \%$. High FDG retention in 
Table 2 Comparison of diagnostic performance between CT and FDG PET/CT in lymph node staging of bladder cancer

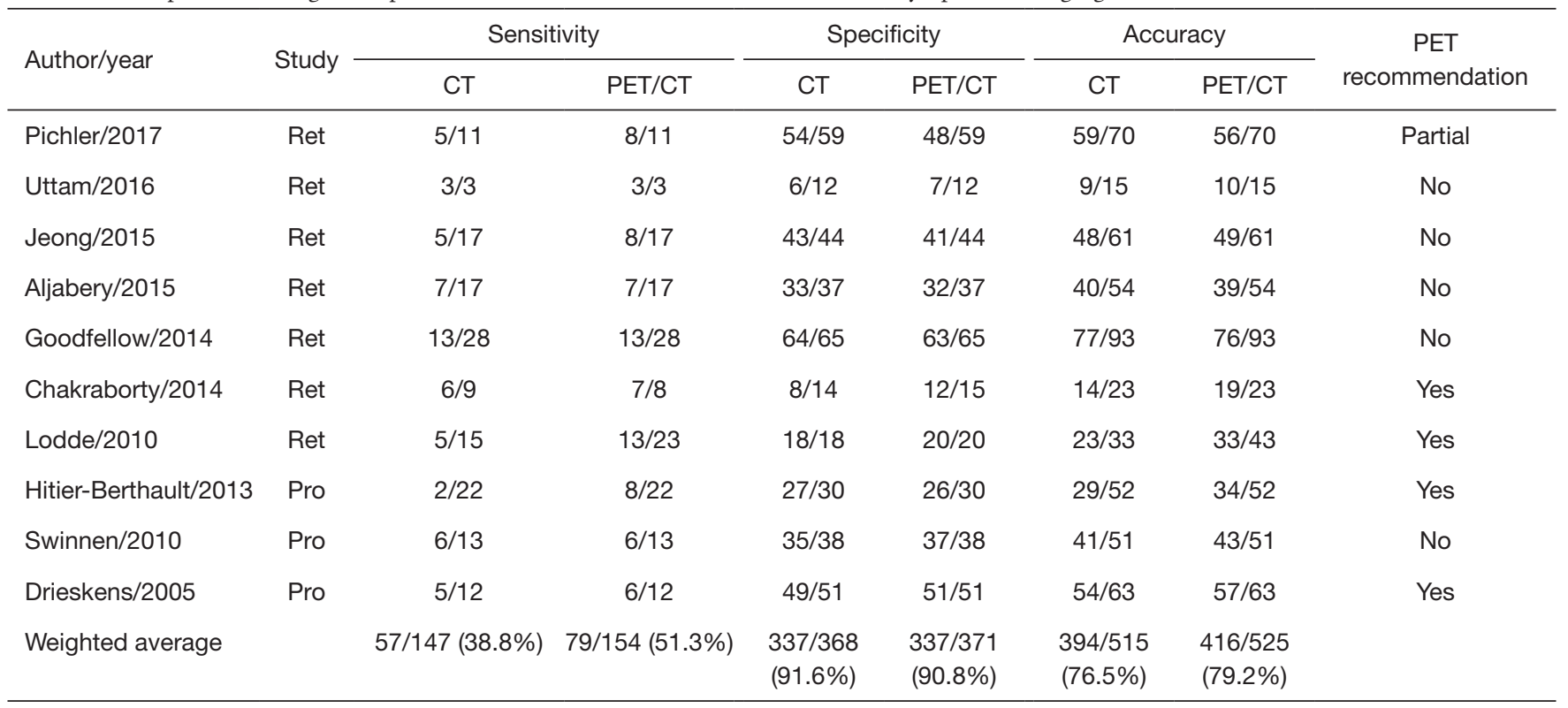

Pro, prospective; Ret, retrospective.

bladder, the extent of LN dissection, small sample size, and study bias could be possible explanations for the low sensitivity. Further research is necessary for substantiating the diagnostic accuracy of FDG PET/CT for this purpose.

As stated earlier, the diagnostic accuracy of CT and MRI was not sufficient in terms of the evaluation of preoperative LN involvement in bladder cancer. Several reports have dealt with the comparison between FDG PET/CT and CT (Table 2), and the results widely varied along studies. A meta-analysis by Soubra et al. (26) reported that the pooled sensitivity of FDG PET/CT was 0.565 (95\% CI: 0.473-0.653); specificity, 0.954 (95\% CI: $0.922-0.976$ ), and those of CT alone was 0.35 (CI: 0.25-0.45); specificity, 0.95 (CI: 0.91-0.98). According to the same study, FDG PET/CT performed better than CT alone; with a pooled sensitivity of $34.5 \%$, CT alone identified $22 \%$ fewer patients with LN metastasis correctly than FDG PET/CT, and FDG PET/CT still performed better in the individual studies. According to the current study, to which additional studies were added, the weighted average sensitivity, specificity, and accuracy of FDG PET/CT and CT were $51.3 \%$, $90.8 \%, 79.2 \%$, and $38.8 \%, 91.6 \%$, and $76.5 \%$, respectively (Table 2). Both FDG PET/CT and CT showed similar high specificity and FDG PET/CT had better sensitivity than $\mathrm{CT}$ in the LN evaluation. Although pooled data showed that FDG PET/CT showed better sensitivity than CT alone, the individual study had contradictory conclusions.
In 4 of the 10 studies enrolled in Table 2, the use of FDG $\mathrm{PET} / \mathrm{CT}$ was advocated for LN evaluation, and 5 studies did not. One study partially advocated the use of FDG $\mathrm{PET} / \mathrm{CT}$ when conventional CT criteria (over $10 \mathrm{~mm}$ in maximum short-axis diameter) were used rather than more sensitive criteria as over $8 \mathrm{~mm}$. At this point, in order to establish the clinical or statistically significant superiority of FDG PET/CT over CT in LN staging, more studies are needed.

\section{Detection of extra-pelvic LNs or distant metastatic lesions using FDG PET/CT}

The main cause of death of bladder cancer is metastases, and metastatic bladder cancer shows poor prognosis. Metastatic lesions can be detected at the time of diagnosis and local recurrence is common after radical cystectomy, and metastasis develops more commonly in MIBC.

Conventional diagnostic methods such as physical exam, CT, and MRI are not currently able to stage metastatic lesions with high accuracy, and their success rate of clinical staging has been reported as $70 \%$ in the literature (34).

Several studies $(15,16,23,29,35)$ found the sensitivity and specificity to be $60 \%, 88 \% ; 77 \%, 97 \% ; 70 \%, 94 \%$; $46 \%, 97 \%$; $57 \%, 100 \%$, respectively in small numbers of MIBC patients (<60 patients) who underwent pathological correlation. The diagnostic performances or detection of 
Table 3 Patient-based diagnostic performances of FDG PET/CT detecting extra-pelvic lesions in the literature

\begin{tabular}{|c|c|c|c|c|c|c|}
\hline Author/year & $\mathrm{N}$ & Sensitivity (\%) & Specificity (\%) & PPV (\%) & NPV (\%) & ACC (\%) \\
\hline Drieskens/2005 & 55 & 60 & 88 & 75 & 79 & 78 \\
\hline Liu/2006 & 55 & 77 & 97 & NR & NR & 84 \\
\hline Kibel/2009 & 43 & 70 & 94 & 78 & 91 & 88 \\
\hline Swinnen/2010 & 50 & 46 & 97 & 85 & 84 & 84 \\
\hline Lodde/2010 & 40 & 57 & 100 & 100 & 80 & 77 \\
\hline Jensen/2011 & 18 & NR & 93 & NR & 87.5 & NR \\
\hline Apolo/2010 & 47 & 87 & 88 & NR & NR & NR \\
\hline Goodfellow/2014 & 93 & 46 & 97 & 87 & 81 & 82 \\
\hline Ozturk/2015 & 79 & 89 & 78 & 90 & 75 & 86 \\
\hline Lu/2012 & NR & 89 & 82 & NR & NR & NR \\
\hline
\end{tabular}

PPV, positive predictive value; NPV, negative predictive value; ACC, accuracy; NR, not reported.

extra-pelvic lesions in the literature were listed in terms of patient-base (Table 3).

A systemic review by $\mathrm{Lu}$ et al. (12) showed sufficient diagnostic accuracy for staging and restaging MIBC and its metastasis (a sensitivity of $89 \%$ and specificity of $82 \%$ ). Other more recent study (36) reported that FDG PET/CT screening in cases of suspected metastatic lesions were retrospectively evaluated and its sensitivity, specificity, accuracy, PPV and NPV were 89\%, 78\%, 90\% (50/55), $75 \%(18 / 24)$, and $86 \%$, respectively, which accorded with Lu et al. (12).

In contrast to Lu et al. (12) and Öztürk et al. (36), Goodfellow et al. reported, in a study with 233 patients with MIBC or high-risk non-MIBC, that FDG PET/CT could detect extra-pelvic metastatic lesions with a sensitivity of $54 \%$ compared to $41 \%$ using CT (20). FDG PET/CT and CT showed similar specificities of $97 \%$ and 98\%, respectively. The addition of a PET scan can improve the detection of distant metastases comparing CT alone. Although FDG PET revealed 5.6\% more patients, PET scans were not routinely recommended because of the small additional clinical advantage and the high financial burden. The pooled diagnostic performance for detecting extrapelvic lesions were summarized from the lesions reported in the literature (Table 4). The pooled sensitivity of FDG PET/CT was demonstrated to be better than that of CT (74.9\% vs. $43.7 \%)$ with similar specificity.

Kibel et al. (16) found 7 patients with occult metastatic disease in a total of 42 patients, and FDG PET/CT influenced the treatment decision prior to radical cystectomy. In the study by Apolo et al. (32) that evaluated 47 patients and 135 individual metastatic lesions, FDGPET/CT led to change the therapeutic plan in $68 \%$ of the patients because it was able to detect $40 \%$ more cases than $\mathrm{CT}$ and MRI imaging conducted together. They concluded that FDG PET/CT provided additional diagnostic information that improved clinical management compared to CT or MRI alone.

Reports advocated that FDG PET/CT revealed extrapelvic metastatic lesions in MIBC with superior sensitivity and specificity (37). Moreover, it has been disclosed that FDG PET/CT provides additional changes in treatment of the disease.

\section{Survival and FDG PET/CT}

Drieskens et al. prospectively revealed that the median survival of PET negative patients was longer than PET negative ones (32.0 vs. 13.5 months) in 55 MIBC or highgrade bladder cancer patients (29). The patients with at least one positive or equivocal lesion were categorized as positive; 12 patients showed PET positivity $(21.8 \%)$ and the remaining 43 showed negativity. PET-negative patients showed $85 \%$ of the 2 -year overall survival and PET-positive did $37 \%$. Although the 6-month overall survival was not reported, the figures could be interpolated from the graph (Table 5), and the 6-month overall survival in PET-positive and negative were 93 and 97 , respectively. 
Table 4 Lesion-based diagnostic performance of FDG PET/CT and CT detecting extra-pelvic lesions*

\begin{tabular}{|c|c|c|c|c|c|c|}
\hline Author/year & \multicolumn{2}{|c|}{ Sensitivity } & \multicolumn{2}{|c|}{ Specificity } & \multicolumn{2}{|c|}{ Accuracy } \\
\hline Drieskens/2005 & $1 / 8$ & $4 / 8$ & $48 / 52$ & $49 / 52$ & $49 / 60$ & $53 / 60$ \\
\hline Liu/2006 & & $10 / 13$ & & $33 / 34$ & & $43 / 47$ \\
\hline Apolo/2010 & & $25 / 31$ & & $15 / 16$ & & $40 / 47$ \\
\hline Ozturk/2015 & & $50 / 56$ & & $18 / 23$ & & $68 / 79$ \\
\hline Soubra/2016 & & $8 / 8$ & & & & $8 / 8$ \\
\hline Pichler/2017** & $7 / 7$ & $7 / 7$ & & & $7 / 7$ & $7 / 7$ \\
\hline Weighted average & $31 / 71(43.7 \%)$ & $134 / 179(74.9 \%)$ & 196/203 (96.6\%) & 262/276 (94.9\%) & $227 / 274(82.8 \%)$ & $396 / 455(87.0 \%)$ \\
\hline
\end{tabular}

*, all lesions, which were remarked as the extra-pelvic lymph nodes and distant metastatic lesions, were pooled; ${ }^{* *}$, Pichler et al. includes secondary malignancies.

Table 5 Overall survival rates in terms of PET positivity

\begin{tabular}{|c|c|c|c|c|c|c|c|}
\hline Author & Patients number & \multicolumn{2}{|c|}{ Median survival (months) } & \multicolumn{2}{|c|}{24 months OS (\%) } & \multicolumn{2}{|c|}{6 months OS (\%) } \\
\hline Drieskens/2005 & 55 & 13.5 & 32.0 & 37 & 85 & $93^{*}$ & $97^{*}$ \\
\hline Kibel/2009 & 42 & $6^{*}$ & $33^{*}$ & 23 & 58 & 63 & 93 \\
\hline Mertens/2014 & 211 & 14 & 50 & $38^{*}$ & $70^{*}$ & $72^{*}$ & $94^{*}$ \\
\hline
\end{tabular}

*, the numbers, which were not reported in detail, were extracted from the survival curve charts in the literature using WebPlotDigitizer (https://automeris.io/WebPlotDigitizer/). +, positive on PET scan; -, negative on PET scan.

In another study by Kiebel et al., PET-positivity was defined visually and semi-quantitatively (16). In total, 42 MIBC patients were enrolled, and PET-positivity was 21.4\% ( $\mathrm{n}=9$ ), while 33 patients showed PET-negativity. The overall survival at 6 and 24 months of PET-positive and negative patients was $63 \%, 23 \%$ and $93 \%, 58 \%$, respectively. The recurrence free survival rates at 6 and 24 months were $50 \%, 0 \%$ and $89 \%, 55 \%$ for PETpositive and negative patients, respectively. The disease specific survival rates at 6 and 24 months were $63 \%, 23 \%$ and $100 \%, 62 \%$ for PET-positive and negative patients, respectively.

PET-positive patients showed significantly poorer results in term of recurrence free survival, disease specific survival rates and overall survival rates than PET-negative patients. The median survival was not reported.

In a recent relatively large study conducted in 2014 by Mertens et al. (38), 211 MIBC patients were evaluated for the relationship of FDG PET/CT results with mortality.
In total, 98 patients $(46 \%)$ showed PET positivity and 113 were negative. The median overall survival rate was 14.0 months with PET positivity and 50.0 months with PET negativity. Overall survival rate between patients with regional or distant PET/CT lesions was not different significantly. The median disease specific survival rates with PET-positive and PET-negative patients were 16.0 and 50.0 months, respectively.

In multivariate survival analyses, a PET-positivity remained an independent predictor of shortened overall survival (38). None of the other variables were associated with overall survival rate, and extravesical lesions on conventional CT was not significant on multivariate survival analysis.

The summary of literature is listed in Table 5. In spite of very limited patient numbers and several weaknesses in study design, the presence of PET-avid lesions was shown to be an independent variable for reduced survival rate without exception. 
A

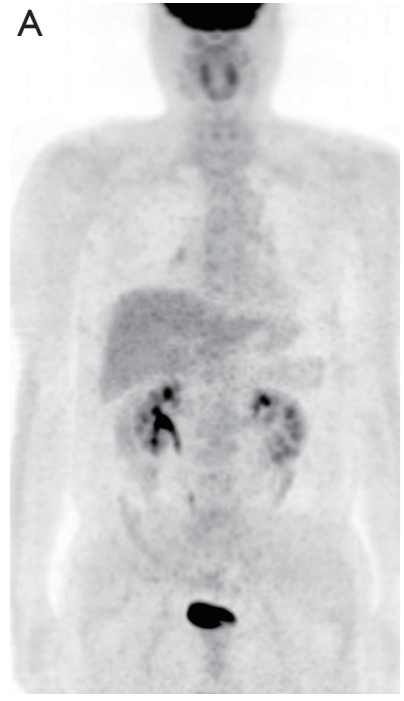

Figure 2 Biodistribution of F-18 FDG (A) and C-11 acetate (B). (A)
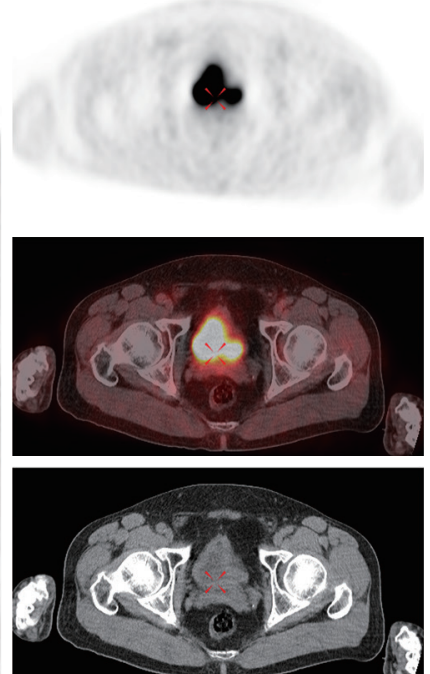

\section{B}
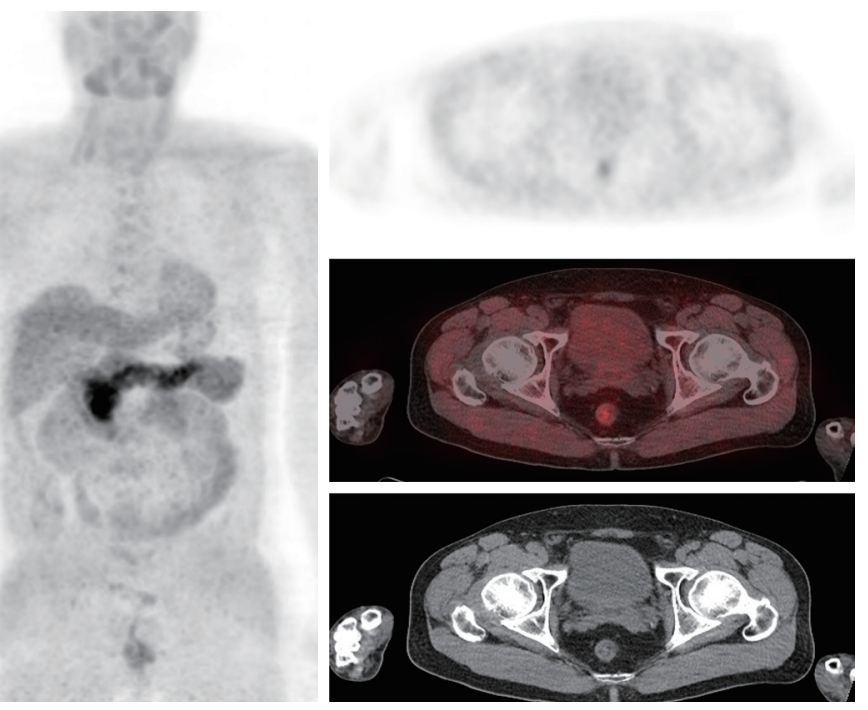

and bladder; (B) physiologic tracer activity is seen in the liver, spleen, and pancreas; excreted activity is seen in the intestines. Myocardial and renal uptake are also shown. Only faint activity is seen in the bladder.

\section{PET/CT using a non-FDG radiotracer}

It is difficult to overcome the innate weakness of FDG for assessing urinary tract tumors. Several C-11 radiotracers with different uptake mechanisms, such as $\mathrm{C}-11$ choline (CHOL), C-11 methionine, and C-11 acetate (ACET), are rarely excreted through the urinary system and are advantageous for the evaluation of the bladder itself (Figure 2). The performance to assess primary lesions $(39,40)$ and regional LN status (41) has been reported in small studies. C-11 radiotracer PET/CT possesses the advantage of providing both anatomic and metabolic information in a single whole-body examination for bladder cancer and involved LNs.

Conventionally, ACET was developed and used for cardiac imaging, and was recently introduced in tumor imaging. The increased ACET uptake and retention in cancer cells is explained by enhanced fatty acid metabolism for cell membranes. As a tumor imaging agent for urinary malignancy, ACET has a critical advantage in that is not excreted into urine. In healthy persons, there is no remarkable ACET uptake except physiologic tracer activity in the liver, spleen, and pancreas.

In three studies $(17,42,43)$, ACET PET/CT were evaluated for their use in bladder cancer and LN metastases, and in one study (44), ACET PET/MR was investigated. In the study by Schöder et al., 17 patients (only 5 patients were over T2, the other were T0-T1) were evaluated (43). Six patients had previously received intravesical BCG therapy and 10 had received neoadjuvant chemotherapy prior to ACET PET/CT. ACET PET/CT correctly identified 80\% of residual bladder cancers (8/10), and two small residual TiS lesions (20\%) were missed, which are attributed to the spatial detection limitation. Of the ACET uptake in the bladder wall, false positive lesions were identified secondary to proliferative cystitis after recent transurethral resection of the bladder or granulomatous cystitis after BCG instillation. The sensitivity and specificity of ACET PET/CT for LN metastasis was $100 \%$ and $87 \%$. Three patients showed true positive uptake in the LN. False positive uptake occurred in $14 \mathrm{LN}$ regions secondary to granulomatous disease after prior intravesical BCG therapy. Vargas et al. (17) reported that ACET accumulated in nine patients within the bladder wall; the ACET accumulation was true-positive in 7 patients and false-positive in 2 patients. Of the remaining ACETnegative 7 patients, 5 had true-negative and 2 had falsenegative results for cancer in the bladder wall. Before ACET PET/CT, 4/16 patients received intravesical BCG treatment, 6/16 did systemic chemotherapy, 3/16 did both, and 3 did neither. Orevi et al. (42) demonstrated in 13 patients that ACET was taken up in all bladder cancers and involved LNs, but it could not detect in situ bladder cancer. ACET PET/CT showed positivity in $10 \mathrm{LNs}$, of which 5 were malignant, which gave a specificity of $50 \%$.

In all three previous studies $(17,42,43)$, ACET PET/ 
Table 6 C-11 acetate PET/CT for bladder cancer detection and lymph node staging

\begin{tabular}{lcccccccc}
\hline Author/year & Purpose & Study & Patients/lesions & Sensitivity (\%) & Specificity (\%) & PPV & NPV & ACC (\%) \\
\hline Vargas/2012 & BC & Pro & 16 & $100(2 / 2)$ & $71(10 / 14)$ & NR & NR & 75 \\
Schoder/2012 & BC & Pro & 16 & $80(8 / 10)$ & $60(3 / 5)$ & NR & NR & 65 \\
& LN & Pro & 17,109 lesions & $100(3 / 3), 100(3 / 3)$ & $64(9 / 14), 87(92 / 106)$ & NR & NR \\
Orevi/2012 & LN & Pro & 14 & $50(5 / 10)$ & & & \\
Salminen/2018 & LN & Pro & 175 lesions & 20 & 96 & & \\
\hline
\end{tabular}

BC, bladder cancer detection; LN, lymph node evaluation; PPV, positive predictive value; NPV, negative predictive value; ACC, accuracy; NR, not reported.

Table 7 Diagnostic performance of C-11 choline PET/CT

\begin{tabular}{lccccccc}
\hline Author/Year & Purpose & Study/N & Sensitivity (\%) & Specificity (\%) & PPV (\%) & NPV (\%) & ACC (\%) \\
\hline Ceci/2015 & Staging & Ret/59 & 59 & 90 & 84 & 81 \\
de Jong/2002 & LN & Pro/18 & 67 & 100 & NR & NR \\
Picchio/2006 & LN* & NR/27 & 62 & 100 & NR & NR \\
Brunocilla/2014 & LN** & Ret/26 & 42 & 84 & NR & NR \\
Maurer/2012 & LN & Pro/44 & 58 & 69 & 39 & 81 \\
Graziani/2015 & LN*** & Ret/25 & 67 & 85 & 80 & 73 \\
& DIS & Ret/25 & 90 & 93 & 90 & 93 \\
\hline
\end{tabular}

${ }^{*}$, study was done after cystectomy; ${ }^{* *}$, patient-based diagnostic performance was calculated; ${ }^{* \star}$, restaging. N, enrolled patients; PPV, positive predictive value; NPV, negative predictive value; ACC, accuracy; Pro, prospective; Ret, retrospective; LN, lymph node evaluation; DIS, distant lesion evaluation; NR, not reported in the article.

CT demonstrated that it has potential, in term of sensitivity and specificity, detecting bladder tumor in comparison with pathology. ACET PET/CT found all MIBC cases and ACET PET/CT seemed to outperform FDG PET/CT due to poor accuracy of FDG for the detection of primary site of bladder cancer. Although these studies showed over-staging affecting accuracy, over-staging seems a less clinically significant than under-staging, which might cause treatment delay. For LN detection, it showed good sensitivity but low specificity. Unfortunately, in the population with prior BCG or transurethral resection of bladder, staging accuracy was negatively affected. ACET PET/CT had the lowest specificity for the detection of nodal metastases due to the high rate of false positive LNs. It remains to be further investigated whether ACET have high positive predictive value in detecting involved LNs.

ACET PET is severely negatively affected by prior neoadjuvant chemotherapy (44). The sensitivity, specificity, and accuracy of ACET PET/MRI detecting MIBC was $1.00,0.69$, and 0.73 in patient base and $0.20,0.96$ and 0.80 in $\mathrm{LN}$ region level, respectively (44). For most imaging modalities including CT and MR, staging accuracy was hampered by previous intravesical and/or systemic chemotherapy. ACET PET was also disappointing until now. It does not seem that ACET PET/MRI is not a satisfactory solution for detecting metastatic pelvic LNs due to limited accuracy after chemotherapy.

MRI, CT, and ACET PET/CT were compared in most previous reports using ACET PET/CT before cystectomy and PLND. These three modalities had similar accuracy for MIBC, although these require further investigation. The pooled data for ACET PET/CT are listed in Table 6.

Other than ACET PET/CT, CHOL PET/CT was evaluated in several studies $(40,45)$ (Table 7). Exogenous choline is phosphorylated into phosphatidylcholine, which is essential component of the cell membrane (46). Cancer is associated with increased cellular proliferation. Following up-regulation of choline kinase, which catalyzes the phosphorylation of choline, provides the rationale using $\mathrm{CHOL}$ as an oncological radiotracer (47). Because $\mathrm{CHOL}$ 
is not excreted into urine, it was reported as a new PET radiotracer for urinary tumors. de Jong et al. (45) showed in their early work that CHOL avidly accumulates in bladder cancer with virtually absent urinary CHOL. Furthermore, no uptake of ACET was observed in premalignant lesions.

The use of CHOL PET/CT, which was mostly evaluated in the literature, is metastatic nodal assessment of MIBC with reference to the pathological specimens. In the study of de Jong et al. (45), CHOL PET/CT showed good performance with a sensitivity of $67 \%$, specificity of $100 \%$, and accuracy of $94 \%$ in 18 patients with bladder cancer. A patient-based analysis by Brunocilla et al. (48) reconfirmed these results with a sensitivity of $42 \%$, specificity of $84 \%$, and accuracy of $73 \%$ in the setting of LN staging (26 patients).

Picchio et al. (40) reported that diagnostic efficacy of CHOL PET/CT was higher than contrast enhanced CT for assessing $\mathrm{LN}$ involvement in bladder cancer staging. On the other hand, Maurer et al. (49) asserted that CHOL PET/CT did not give additional diagnostic benefit during preoperative LN staging, in comparison with contrast enhanced CT. 27\% patients revealed LN metastasis (12/44), and the sensitivity, specificity, PPV, NPV, and accuracy for CHOL PET/CT were reported as $58 \%, 66 \%, 39 \%$, $81 \%$, and $64 \%$, respectively, analyzing on patient-base; and for CT those were $75 \%, 56 \%, 39 \%, 86 \%$, and $61 \%$, respectively.

CHOL PET/CT may supply more diagnostic information in preoperative $\mathrm{LN}$ staging of bladder cancer patients and be considered a useful tool for restaging patients suspecting bladder cancer relapse. In bladder cancer population, CHOL PET/CT had a sensitivity, specificity, PPV, NPV and accuracy of $59 \%, 90 \%, 71 \%, 84 \%$, and $81 \%$, respectively (50). CHOL PET/CT is helpful for the restaging of $\mathrm{BC}$ patients suspected of relapse. In particular, CHOL PET/CT offered appropriate sensitivity, specificity, accuracy, PPV, and NPV for the identification of LN or distant metastases. CHOL PET/CT showed a sensitivity, specificity, accuracy, PPV, and NPV of $66.7 \%, 84.6 \%, 76 \%, 80 \%$, and $73.3 \%$ for local relapse and $90 \%, 93.3 \%, 92 \%, 90 \%$, and $93.3 \%$ for LNs and distant relapse, respectively (51).

In a prospective trial, neither CT nor CHOL PET/CT were able to sufficiently predict overall survival or cancer specific death in bladder cancer patients treated with radical cystectomy, although trends and moderately increased hazard ratios could be demonstrated without significant differences between CT and CHOL PET/CT (52). In summary, CHOL PET/CT demonstrates good sensitivity and specificity detecting LN metastasis in bladder cancer with some discrepancy in the results. It could be helpful for restaging bladder cancer patients with a high risk of relapse after primary therapy.

The $\mathrm{C}-11$ radiotracer has a disadvantage compared to FDG because of its need for an expensive on-site cyclotron and its short physical half-life of 20 minutes, whereas, CHOL and ACET have the advantage of minimal urinary excretion and thus a higher sensitivity for the detection of local relapse compared to FDG (40). The tumoral uptake of ACET and CHOL are similar in bladder cancer. The mean tumor-to-background ratios (TBR) for the bladder tumor on ACET and CHOL were 4.5 (range, 1.9-8.7) and 6.0 (range, 2.0-12.7), respectively, and those for LNs were 9.8 (range, 2.6-44.0) and 13.2 (range, 4.3-50.0), respectively (42). SUVs of CHOL accumulation were slightly higher in bladder cancers, which was not statistically significant, than ACET whereas ACET accumulates more in LNs than CHOL. TBR of CHOL was not significantly higher than ACET for bladder cancers but significantly higher for LNs. The higher TBR for CHOL and ACET may be due to a lower background uptake rather than a higher tumoral uptake, and there may be an advantage for $\mathrm{C}-11$ radiotracer imaging of bladder cancer and metastatic LNs. It should be noted that these results relate only to CHOL and ACET, as the corresponding FDG are excreted into urine and cannot reveal primary bladder cancers.

\section{Conclusions}

Recently, FDG and non-FDG PET/CT imaging have been increasingly studied as additive tools for the management of select bladder cancer patients. Several retrospective and prospective studies supported its superiority to CT in the staging of $\mathrm{LN}$ and extra-pelvic metastases, as well as re-staging, although there were some discrepant results. There are promising results for the use of new non-FDG radiotracer as an alternative to FDG, which causes a high degree of difficulty in the assessment of the genitourinary tract, despite its limited availability. This short review outlined the role of PET/CT imaging in bladder cancer.

\section{Acknowledgments}

Funding: This research was supported by National Cancer Center, Korea Grant (NCC-1810201). 


\section{Footnote}

Provenance and Peer Review: This article was commissioned by the Guest Editors (Ja Hyeon Ku, Ho Kyung Seo, Seok Ho Kang) for the series "Muscle-Invasive Bladder Cancer" published in Translational Andrology and Urology. The article has undergone external peer review.

Conflicts of Interest: The author has completed the ICMJE uniform disclosure form (available at http://dx.doi. org/10.21037/tau.2020.03.31). The series "Muscle-Invasive Bladder Cancer" was commissioned by the editorial office without any funding or sponsorship. The author has no other conflicts of interest to declare.

Ethical Statement: The author is accountable for all aspects of the work in ensuring that questions related to the accuracy or integrity of any part of the work are appropriately investigated and resolved.

Open Access Statement: This is an Open Access article distributed in accordance with the Creative Commons Attribution-NonCommercial-NoDerivs 4.0 International License (CC BY-NC-ND 4.0), which permits the noncommercial replication and distribution of the article with the strict proviso that no changes or edits are made and the original work is properly cited (including links to both the formal publication through the relevant DOI and the license). See: https://creativecommons.org/licenses/by-nc-nd/4.0/.

\section{References}

1. Kaufman DS, Shipley WU, Feldman AS. Bladder cancer. Lancet 2009;374:239-49.

2. Lee ST, Lawrentschuk N, Scott AM. PET in prostate and bladder tumors. Semin Nucl Med 2012;42:231-46.

3. Alfred Witjes J, Lebret T, Comperat EM, et al. Updated 2016 EAU Guidelines on Muscle-invasive and Metastatic Bladder Cancer. Eur Urol 2017;71:462-75.

4. Spiess PE, Agarwal N, Bangs R, et al. Bladder Cancer, Version 5.2017, NCCN Clinical Practice Guidelines in Oncology. J Natl Compr Canc Netw 2017;15:1240-67.

5. Nayak B, Dogra PN, Naswa N, et al. Diuretic 18F-FDG PET/CT imaging for detection and locoregional staging of urinary bladder cancer: prospective evaluation of a novel technique. Eur J Nucl Med Mol Imaging 2013;40:386-93.

6. Yildirim-Poyraz N, Ozdemir E, Uzun B, et al. Dual phase 18F-fluorodeoxyglucose positron emission tomography/ computed tomography with forced diuresis in diagnostic imaging evaluation of bladder cancer. Rev Esp Med Nucl Imagen Mol 2013;32:214-21.

7. Zhang $H$, Xing $W$, Kang Q, et al. Diagnostic value of $[18 F]$ FDG-PET and PET/CT in urinary bladder cancer: a meta-analysis. Tumour Biol 2015;36:3209-14.

8. Paik ML, Scolieri MJ, Brown SL, et al. Limitations of computerized tomography in staging invasive bladder cancer before radical cystectomy. J Urol 2000;163:1693-6.

9. Herr HW, Dotan Z, Donat SM, et al. Defining optimal therapy for muscle invasive bladder cancer. J Urol 2007;177(2):437-43.

10. Kim JK, Park SY, Ahn HJ, et al. Bladder cancer: analysis of multi-detector row helical CT enhancement pattern and accuracy in tumor detection and perivesical staging. Radiology 2004;231:725-31.

11. Jager GJ, Barentsz JO, Oosterhof GO, et al. Pelvic adenopathy in prostatic and urinary bladder carcinoma: MR imaging with a three-dimensional TI-weighted magnetization-prepared-rapid gradient-echo sequence. AJR Am J Roentgenol 1996;167:1503-7.

12. Lu YY, Chen JH, Liang JA, et al. Clinical value of FDG PET or PET/CT in urinary bladder cancer: a systemic review and meta-analysis. Eur J Radiol 2012;81:2411-6.

13. Anjos DA, Etchebehere EC, Ramos CD, et al. 18F-FDG PET/CT delayed images after diuretic for restaging invasive bladder cancer. J Nucl Med 2007;48:764-70.

14. Kosuda S, Kison PV, Greenough R, et al. Preliminary assessment of fluorine-18 fluorodeoxyglucose positron emission tomography in patients with bladder cancer. Eur J Nucl Med 1997;24:615-20.

15. Swinnen G, Maes A, Pottel H, et al. FDG-PET/CT for the preoperative lymph node staging of invasive bladder cancer. Eur Urol 2010;57:641-7.

16. Kibel AS, Dehdashti F, Katz MD, et al. Prospective study of $[18 \mathrm{~F}]$ fluorodeoxyglucose positron emission tomography/ computed tomography for staging of muscle-invasive bladder carcinoma. J Clin Oncol 2009;27:4314-20.

17. Vargas HA, Akin O, Schoder H, et al. Prospective evaluation of MRI, (1)(1)C-acetate PET/CT and contrast-enhanced CT for staging of bladder cancer. Eur J Radiol 2012;81:4131-7.

18. Aljabery F, Lindblom G, Skoog S, et al. PET/CT versus conventional CT for detection of lymph node metastases in patients with locally advanced bladder cancer. BMC Urol 2015;15:87.

19. Chakraborty D, Mittal BR, Kashyap R, et al. Role of fluorodeoxyglucose positron emission tomography/ 
computed tomography in diagnostic evaluation of carcinoma urinary bladder: comparison with computed tomography. World J Nucl Med 2014;13:34-9.

20. Goodfellow H, Viney Z, Hughes P, et al. Role of fluorodeoxyglucose positron emission tomography (FDG PET)-computed tomography (CT) in the staging of bladder cancer. BJU Int 2014;114:389-95.

21. Jensen TK, Holt P, Gerke O, et al. Preoperative lymphnode staging of invasive urothelial bladder cancer with $18 \mathrm{~F}$-fluorodeoxyglucose positron emission tomography/ computed axial tomography and magnetic resonance imaging: correlation with histopathology. Scand J Urol Nephrol 2011;45:122-8.

22. Jeong IG, Hong S, You D, et al. FDG PET-CT for lymph node staging of bladder cancer: a prospective study of patients with extended pelvic lymphadenectomy. Ann Surg Oncol 2015;22:3150-6.

23. Lodde M, Lacombe L, Friede J, et al. Evaluation of fluorodeoxyglucose positron-emission tomography with computed tomography for staging of urothelial carcinoma. BJU Int 2010;106:658-63.

24. Pichler R, De Zordo T, Fritz J, et al. Pelvic Lymph Node Staging by Combined (18)F-FDG-PET/CT Imaging in Bladder Cancer Prior to Radical Cystectomy. Clin Genitourin Cancer 2017;15:e387-e395.

25. Rouanne M, Girma A, Neuzillet Y, et al. Potential impact of 18F-FDG PET/CT on patients selection for neoadjuvant chemotherapy before radical cystectomy. Eur J Surg Oncol 2014;40:1724-30.

26. Soubra A, Hayward D, Dahm P, et al. The diagnostic accuracy of 18F-fluorodeoxyglucose positron emission tomography and computed tomography in staging bladder cancer: a single-institution study and a systematic review with meta-analysis. World J Urol 2016;34:1229-37.

27. Uttam M, Pravin N, Anish B, et al. Is [F-18]fluorodeoxyglucose FDG-PET/CT better than CT alone for the preoperative lymph node staging of muscle invasive bladder cancer? Int Braz J Urol 2016;42:234-41.

28. Bachor R, Kotzerke J, Reske SN, et al. Lymph node staging for urinary bladder carcinoma with positron emission tomography (PET). Der Urologe 1999;38:46-50.

29. Drieskens O, Oyen R, Van Poppel H, et al. FDG-PET for preoperative staging of bladder cancer. Eur J Nucl Med Mol Imaging 2005;32:1412-7.

30. Hitier-Berthault M, Ansquer C, Branchereau J, et al. 18 F-fluorodeoxyglucose positron emission tomographycomputed tomography for preoperative lymph node staging in patients undergoing radical cystectomy for bladder cancer: a prospective study. Int J Urol 2013;20:788-96.

31. Girard A, Rouanne $M$, Taconet $S$, et al. Integrated analysis of (18)F-FDG PET/CT improves preoperative lymph node staging for patients with invasive bladder cancer. Eur Radiol 2019;29:4286-93.

32. Apolo AB, Riches J, Schoder H, et al. Clinical value of fluorine-18 2-fluoro-2-deoxy-D-glucose positron emission tomography/computed tomography in bladder cancer. J Clin Oncol 2010;28:3973-8.

33. Ha HK, Koo PJ, Kim SJ. Diagnostic Accuracy of F-18 FDG PET/CT for Preoperative Lymph Node Staging in Newly Diagnosed Bladder Cancer Patients: A Systematic Review and Meta-Analysis. Oncology 2018;95:31-8.

34. Sternberg CN, Pansadoro V, Calabro F, et al. Can patient selection for bladder preservation be based on response to chemotherapy? Cancer 2003;97:1644-52.

35. Liu IJ, Lai YH, Espiritu JI, et al. Evaluation of fluorodeoxyglucose positron emission tomography imaging in metastatic transitional cell carcinoma with and without prior chemotherapy. Urol Int 2006;77:69-75.

36. Öztürk H. Detecting Metastatic Bladder Cancer Using (18) F-Fluorodeoxyglucose Positron-Emission Tomography/ Computed Tomography. Cancer Res Treat 2015;47:834-43.

37. Bouchelouche K, Turkbey B, Choyke PL. PET/CT and MRI in Bladder Cancer. J Cancer Sci Ther 2012;S14.

38. Mertens LS, Mir MC, Scott AM, et al. $18 \mathrm{~F}$-fluorodeoxyglucose--positron emission tomography/ computed tomography aids staging and predicts mortality in patients with muscle-invasive bladder cancer. Urology 2014;83:393-8.

39. Gofrit ON, Mishani E, Orevi M, et al. Contribution of 11C-choline positron emission tomography/computerized tomography to preoperative staging of advanced transitional cell carcinoma. J Urol 2006;176:940-4; discussion 944.

40. Picchio M, Treiber U, Beer AJ, et al. Value of $11 \mathrm{C}$-choline PET and contrast-enhanced CT for staging of bladder cancer: correlation with histopathologic findings. J Nucl Med 2006;47:938-44.

41. Kim SJ, Koo PJ, Pak K, et al. Diagnostic accuracy of $\mathrm{C}-11$ choline and $\mathrm{C}-11$ acetate for lymph node staging in patients with bladder cancer: a systematic review and meta-analysis. World J Urol 2018;36:331-40.

42. Orevi M, Klein M, Mishani E, et al. 11C-acetate PET/CT in bladder urothelial carcinoma: intraindividual comparison with 11C-choline. Clin Nucl Med 2012;37:e67-72.

43. Schöder H, Ong SC, Reuter VE, et al. Initial results with 
(11)C-acetate positron emission tomography/computed tomography (PET/CT) in the staging of urinary bladder cancer. Mol Imaging Biol 2012;14:245-51.

44. Salminen A, Jambor I, Merisaari H, et al. (11)C-acetate PET/MRI in bladder cancer staging and treatment response evaluation to neoadjuvant chemotherapy: a prospective multicenter study (ACEBIB trial). Cancer Imaging 2018;18:25.

45. de Jong IJ, Pruim J, Elsinga PH, et al. Visualisation of bladder cancer using (11)C-choline PET: first clinical experience. Eur J Nucl Med Mol Imaging 2002;29:1283-8.

46. Zeisel SH. Dietary choline: biochemistry, physiology, and pharmacology. Annu Rev Nutr 1981;1:95-121.

47. Katz-Brull R, Degani H. Kinetics of choline transport and phosphorylation in human breast cancer cells; NMR application of the zero trans method. Anticancer Res 1996;16:1375-80.

48. Brunocilla E, Ceci F, Schiavina R, et al. Diagnostic accuracy of (11)C-choline PET/CT in preoperative lymph node staging of bladder cancer: a systematic comparison with contrast-enhanced CT and histologic findings. Clin Nucl Med 2014;39:e308-12.

49. Maurer T, Souvatzoglou M, Kubler H, et al. Diagnostic efficacy of [11C]choline positron emission tomography/ computed tomography compared with conventional computed tomography in lymph node staging of patients with bladder cancer prior to radical cystectomy. Eur Urol 2012;61:1031-8.

50. Ceci F, Bianchi L, Graziani T, et al. 11C-choline PET/ CT and bladder cancer: lymph node metastasis assessment with pathological specimens as reference standard. Clin Nucl Med 2015;40:e124-8.

51. Graziani T, Ceci F, Lopes FL, et al. 11C-choline PET/ CT for restaging of bladder cancer. Clin Nucl Med 2015;40:e1-5.

52. Maurer T, Horn T, Souvatzoglou M, et al. Prognostic value of 11C-choline PET/CT and CT for predicting survival of bladder cancer patients treated with radical cystectomy. Urol Int 2014;93:207-13.

Cite this article as: Kim SK. Role of PET/CT in muscleinvasive bladder cancer. Transl Androl Urol 2020;9(6):2908-2919. doi: $10.21037 /$ tau.2020.03.31 